\title{
Feeding back on ingestion
}

New research has identified a population of prodynorphin-expressing

Direct stimu-

lation of $\mathrm{PB}^{\text {Pdyn }}$ neurons

potently

suppressed

food and water intake in mice neurons in the parabrachial nucleus $\left(\mathrm{PB}^{\text {Pdyn }}\right.$ neurons) in mice that monitors ingestion and mediates a negative feedback control upon eating or drinking. This feedback control relies on mechanosensory signals from the upper gastrointestinal tract, ranging from the mouth to the stomach, according to the findings published in Nature.

"How do we stop eating when we feel full? As we experience on a daily basis, negative sensory feedback from the digestive tract to the brain is critical for limiting appetite," explains author Sung-Yon Kim. However, little is known about the specific mechanism by which mechanosensory feedback signalling from the gut reduces appetite. Although neurons in the vagus nerve that sense distention in the stomach and intestine have been identified, no subpopulations of neurons in the brain that encode gut mechanosensation have yet been described. Using a variety of techniques, including deep-brain calcium imaging experiments at the population and single-cell levels, Kim and colleagues sought to identify and characterize these neurons in mice.

First, the investigators assessed real-time activity of $\mathrm{PB}^{\text {Pdyn }}$ neurons using fibre photometry and confirmed

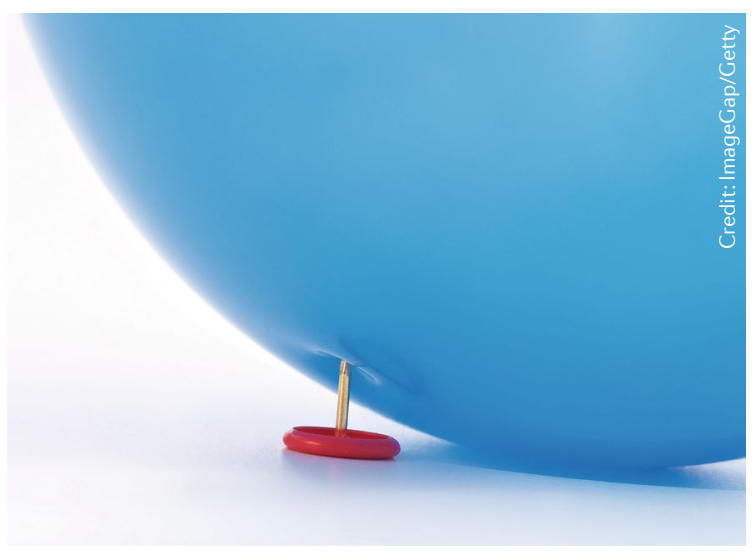

that these neurons were activated in mice upon ingestion of a variety of liquids (including liquid food and water) and solids (hydrogel and chow). Indeed, the amplitude of the neuronal response was positively correlated with the ingestion rate, implicating mechanosensory signals relayed from the gastrointestinal tract. Diverse mechanical stimuli to different parts of the gastrointestinal tract, including the tongue, oesophagus and stomach, robustly activated $\mathrm{PB}^{\text {Pdyn }}$ neurons.

Notably, mechanical stimulation of both the duodenum and proximal colon did not evoke the same responses. Strikingly, single-cell calcium imaging revealed that signals arising from different parts of the gastrointestinal tract converged on individual $\mathrm{PB}^{P d y n}$ neurons, indicating that these neurons might be a hub for integration.

$\mathrm{PB}^{\text {Pdyn }}$ neurons were found to be anatomically connected to the digestive periphery via transmission through cranial and spinal pathways. Importantly, the researchers mapped the input pathways of $\mathrm{PB}^{\text {Pdyn }}$ neurons and determined that the vagus nerve in particular conveys signals arising from gastric distention to $\mathrm{PB}^{\text {Pdyn }}$ neurons; vagotomy abolished the responses of $\mathrm{PB}^{\text {Pdyn }}$ neurons to gastric distention.

Using both chemogenetic and optogenetic tools, it was shown that $\mathrm{PB}^{\text {Pdyn }}$ neurons transmit sustained appetite-suppressing signals to discourage ingestion, particularly to suppress the initiation of feeding and drinking, and are required for negative feedback control of ingestion. Direct stimulation of $\mathrm{PB}^{\text {Pdyn }}$ neurons potently suppressed food and water intake in mice by reducing the number of bouts of intake as opposed to the duration of the bout. By contrast, inhibition of $\mathrm{PB}^{\text {Pdyn }}$ neurons increased intake of chow food in food-deprived mice and both water and hypertonic saline in dehydrated mice. Notably, inhibition of $\mathrm{PB}^{\text {Pdyn }}$ neurons did not affect water or saline intake in mice that were adequately hydrated already, implying that the reduced activity of $\mathrm{PB}^{\text {Pdyn }}$ neurons causes overconsumption only when the appetite already exists rather than stimulating appetite itself.

Finally, the researchers observed that $\mathrm{PB}^{\text {Pdyn }}$ neurons densely innervated many areas of the brain that are critical for intake behaviours. These innervations included projections to the paraventricular hypothalamus, which the investigators suggest at least partially mediates the suppression of ingestion as a downstream projection target of $\mathrm{PB}^{\text {Pdyn }}$ neurons.

"Our findings collectively provide a mechanism by which mechanosensation from the upper digestive tract is integrated and transduced to a negative sensory feedback signal that reduces appetite," states Kim. "Notably, the molecular identity of the mechanotransduction channel for monitoring ingestion remains unknown," he says, adding that mechanosensitive Piezo, ASIC and TRP ion channels, which have been shown to be expressed in the gastrointestinal tract, are possible candidates.

The researchers plan to further explore how gastrointestinal tract distension controls ingestion, a key question in physiology. "We are interested in identifying the circuit node that integrates appetite-suppressing signals produced from gut mechanosensation with the internal need state and the mechanisms underlying this integration," says Kim, concluding that these identified circuits will be important candidates for developing novel therapeutic strategies for obesity and other metabolic disorders.

Katrina Ray

ORIGINAL ARTICLE Kim, D. -Y. et al. A neural circuit mechanism for mechanosensory feedback control of ingestion. Nature https://doi.org/ 10.1038/s41586-020-2167-2 (2020) 